\title{
General Model of Current Course-Selection System
}

\section{Zhouhongyi Yang}

International School of Beijing University of Posts and Telecommunications, Beijing, 100876, China 2014213073@bupt.edu.cn

Keywords: Information manage system, Course-selection system.

\begin{abstract}
Since the theory of campus informationization proposed in MIT, the information management system, as the most effective method used in campus, has become universal in the worldwide. Many China universities have developed or purchased its own integrated educational management system to satisfy the requirements of course management. In this paper, we focus on analyzing the general model of course-selection system in the step of requirement specification, framework identification and operation flow formulation, which will lead to a better understanding thorough making a conclusion of current course-selection system and having a discussion to its future.
\end{abstract}

\section{Introduction}

The formation and development of course-selection system, though it is an emerging technological application rising recently, has covered a period of time. Before the popularization of computer and Internet in 1980s, traditional course-selection is an intricate and complicated process: students would get a paper offered by dean's office to know what kind of courses were elective, then went to a designated classroom to first enroll a course. After collecting all the information, a timetable was formulated by dean's office [1]. In this case, due to the artificial arrangement of the timetable, it was likely to happen that students who come after hard to enroll the ideal courses. What' worse, with the expansion of school scale and business growth, this mode cannot meet with the educational demand [2].

However, since the education reform and development of the computer technology in late 1990s, Education Department of China approved four colleges including Tsinghua University to carry out network education, which emphasized on developing educational administration management system based on campus network [3].

With one well-known concept, which is known as campus informationization now, released by MIT, a mature campus information management platform was formed [4]. Another concept which is known as Management Information System was released by Gordon B.Davis in 1960s, who considered it as a system which uses computer hardware and software to analyze plan control and decision making [5].

With the help of internet and MIS, inchoate system was given birth in Chinese universities in late 1990s. Applying information technology into higher education field in developed country had laid foundation for course selection system. After few years, most campus in China realized the informationization through the management system in school, students can finish enrollment, select courses, and enroll tests through the platform.

\section{General Model}

\subsection{Requirement specification}

The system should realize the function of reallocating teaching resources in order to achieve a higher resources utility. In a sample requirement specification of UESTC, the system can meet with various demands from teachers, students and dean's office [6]. After analyzing and concluding many course-selection systems from different universities, the overview requirements for system can be specified into the following modules (shown in fig1): 
1. Student module: This module aims at enabling students to search courses they want and modify their personal information and courses information while meeting with the basic needs of course selecting. Hence, the module should include functions of course selection, course inquiry, course management, and grades inquiry and information modification.

2. Teacher module: In this module, it should have some functions that allow teachers to manage courses they are responsible for, such as function of course set up, course management, student information management, grades management and information modification.

3. Dean's module: In order to improve the efficiency, there should have another module to support teacher module and student module in the way of collecting courses information from both teachers and students to make an overall timetable for the teaching plan. This module should have the function of course management, timetable set up, student information management and teacher information management.

4. Admins module: Every system should have administrator, admin module should include user management, password management and jurisdiction management.

\subsection{Identifying Framework}

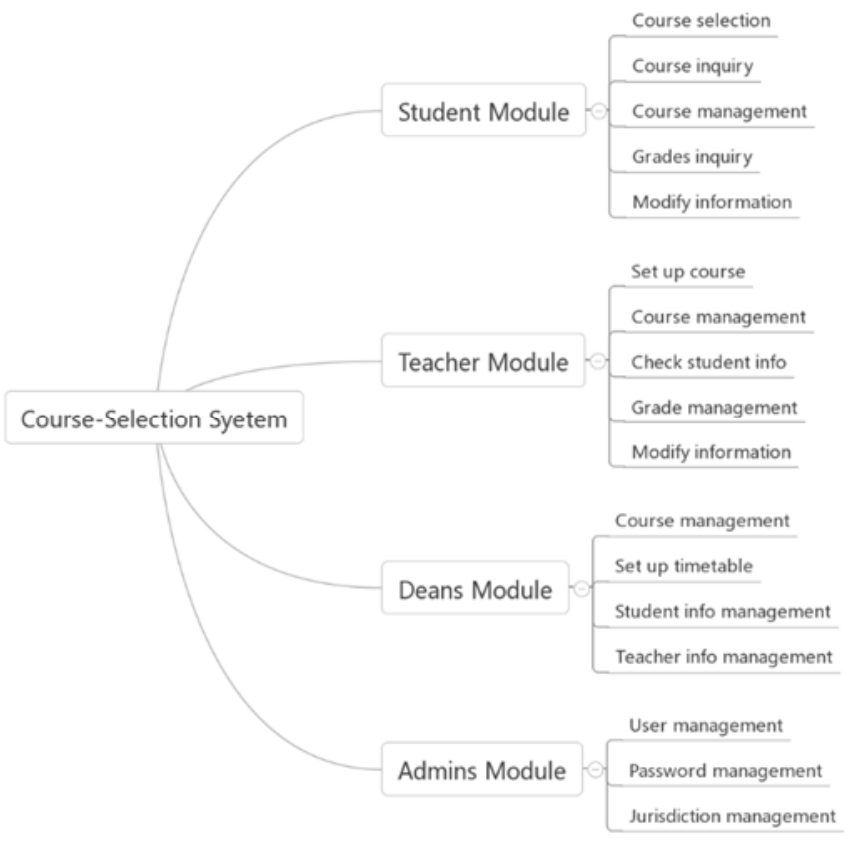

Fig1. Course Selection System

\subsubsection{C/S Framework Introduction}

C/S framework, a well-known structure for software system, consists of client part and server part, can make full use of the hardware of both client devices and servers, avoiding the condition of being overloaded. Most business logic and interface are finished in client and through interacting with server to satisfy the system needs.

Using C/S framework, the operation and user interface can be designed well organized, and will have a quicker response with only one layer between client and server to interact. However, this also lead to the condition of high maintenance costs that all clients should change when updates.

\subsubsection{B/S Framework Introduction}

Browser/Server framework, just as its name, is consists of browser, WebApp and database server. Browser can realize the function of display and interact with user, while WebApp take charge of business logic. In this framework, user does not need to install client if their computer have a web browser. What's more, unlike C/S framework, system with B/S framework just need to update server instead of updating all the clients. However, B/S framework also remains some foibles [7]. For example, it does not performed well when facing the cross-browser. Additionally, it is difficult for $\mathrm{B} / \mathrm{S}$ framework to achieve the property of C/S framework. What's the most important, improving speed ability and safety ability of a B/S framework cost a lot. Both C/S and B/S framework are shown in fig2. 


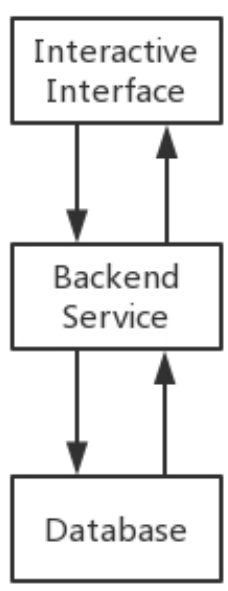

CIS framework

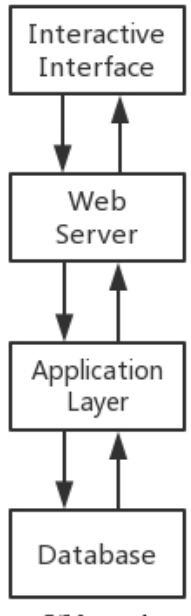

B/S framework

\subsubsection{Identify Framework}

Fig 2. C/S \& B/S framework

$\mathrm{B} / \mathrm{S}$ framework and C/S framework have different properties, we can use mixed framework to design the system. The module suitable for $\mathrm{C} / \mathrm{S}$ framework should have the characteristics such as high demand for safety ability and respond speed ability, while the module suitable for B/S framework does not have fixed location to use. So we can divide modules into two parts, which student module and teacher module can be designed by B/S framework, deans' module and admin module can be designed by $\mathrm{C} / \mathrm{S}$ framework.

\section{Operation flow}

In this section, we take every condition and requirement into consideration to design system. Course-selection system is used for student to have a better experience of course-selection, consisting of four parts. We exemplify an operation flow of course selection function in Student module, one of the most essential part of this system, to show how the system works. A flow chart is given in fig3.

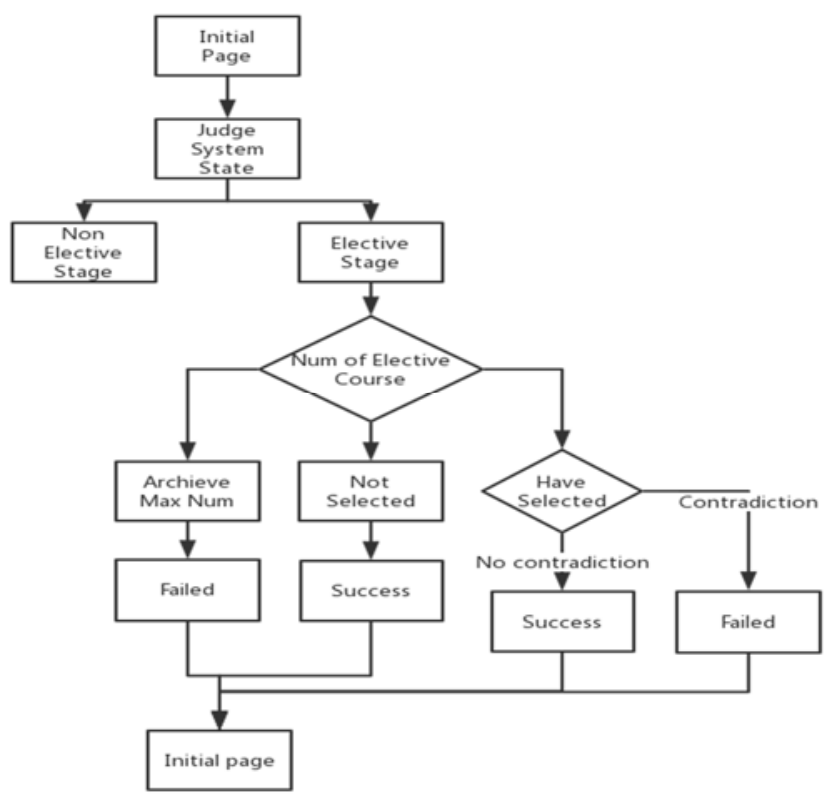

Fig3. Flow chart of course selection

To begin with, we should analysis both the functional requirements and non-functional requirements of this module. The functional requirement of this module, without doubt, is to provide the service for students selecting courses. When it comes to non-functional requirements, we should 
take some restrictions into consideration. As a student, he could only do the operation of selecting courses in a specified time period, which is also called "Elective Stage". There's no doubt that a student cannot choose two class that starts at the same time. In addition, a student should have an upper limit of the number of class that could be chosen.

After analyzing the module requirements, the operation flow should be decided for this module. First, while a student log in the system, there should exist a decision mechanism that judge whether it is during the session for course-selection. If it is the stage for course-selection, the selecting page will be shown for student, where student can glance overall courses information and choose the one suitable for him. Then the system should judge the operation is valid or not. If student cannot select more courses or the time of selected course have contradiction, the operation will be consider invalid and the page will back to the previous one, otherwise, the operation is valid and the result will be saved.

\section{Discussion}

With the rapid development of computer technology and network technique, informationization is widely spread, not only in universities, but also in some senior high school. Course-selection system, as an essential part of integrated information management system, it absolutely meet the demand of campus informationization and greatly improve the resource utility.

However, there still remains some problems and defects to be improved. In some research [8] , the opinions are hold that due to the expansion of the scale of colleges and Universities and increase in number of teachers and students, there are some foibles in the aspect of security, response spend and accuracy. For example, some campus may be far away from the main campus, which makes a hidden danger in data security. And the large scale will cause the phenomenon that most student use the system in a certain date simultaneously that brings a huge pressure to the server. In addition, in order to consummate the process of campus informationization, the course-selection system should be able to interact with other information management system in campus, such as the library system to enable student to use resources from library and search some booking information more convenient.

There should be countless and comprehensive work to be done to realize the campus informationization.

\section{References}

[1]. Yunpeng Bai. Design and implementation of online course selection system based on B/S mode. Jilin. Jilin University

[2]. Lijun Liu. Research and development of student information management system. Journal of Taizhou Polytechnic Institute. 2005(02): 63-66.

[3]. Lifang Liu, Yanhong Wang. A review of the research on the development of curriculum management system in China. The Chinese Journal of ICT in Education. 2010(03): 13-16.

[4]. Liying He. The design and implementation of university student information management system. Chongqing. University of Electronic Science and Technology of China.

[5]. Shiqing Yang. Discovery on Students information management system of Network Base. Wuhan. Wuhan University of Technology.

[6]. Qiang Shao. Design and implementation of the college student's course-selecting system. Chongqing. University of Electronic Science and Technology of China.

[7]. Hongwei Zhao, Changmin Qin. Research on software deisgn based on B/S 3-Tier structure. Research and Exploration in Laboratory. 2011(07): 64-66.

[8]. Zongzhen Gao, Study and implement on Online Course Selection System Based on Load Balancing. Qingdao. Ocean University of China. 